\title{
Actualización en los protocolos de extracción dental en pacientes anticoagulados
}

\section{Update in dental procedures in patients receiving oral anticoagulated therapy}

\author{
Ripollés de Ramón J*, Gómez Font R**, Muñoz-Corcuera $M^{*}$, \\ Bascones Martínez A***
}

\section{RESUMEN}

Las intervenciones de cirugía oral y las simples extracciones dentales pueden ser objeto de complicaciones inmediatas y mediatas desde un punto de vista hemorrágico para el profesional que interviene sobre pacientes en tratamiento con anticoagulantes orales. Los protocolos clínicos publicados en relación al enfoque terapéutico a seguir en estos casos se basa en dos vertientes: la que postula una disminución de la ingesta farmacológica del anticoagulantes y sustitución por heparina o bien aquella que preconiza su mantenimiento y el control del INR junto con unas medidas hemostáticas específicas del proceso. Nuestro propósito es evaluar los protocolos descritos en la literatura y por las sociedades médicas con el fin de iniciar unas pautas aclaratorias actualizadas. Palabras clave: Terapia anticoagulante, hemostasia, protocolo, extracción dental.

\section{SUMMARY}

Oral surgery and easy dental extractions could be potential mediate or immediate clinical hemorrhagic complications that dental clinicians may encounter when treating patients on anticoagulant oral medication. The clinical protocols published regarding which therapeutic procedure to follow when dealing with these patients are based on two approaches: One that postulates a decrease of the pharmacological ingestion of the anticoagulants and their substitution with heparin; another which recommends their maintenance and the control of INR (international normalized ratio) together with a few haemostatic specific procedures.

Our aim is, therefore, to evaluate the protocols described in the literature in order to establish a few explanatory updated guidelines for the medical associations.

Key words: Anticoagulant therapy, haemostasia, protocol, dental extraction.

Fecha de recepción: 11 de mayo de 2012.

Aceptado para publicación: 10 de junio de 2012.

* Doctor en Odontología. Colaborador del Departamento de Estomatología III. Facultad Odontología UCM.

** Profesor asociado Estomatología III, Facultad de Odontología. UCM.

*** Catedrático Medicina bucal y Periodoncia. Facultad Odontología UCM.

Ripollés de Ramón J, Gómez Font R, Muñoz-Corcuera M, Bascones Martínez A. Actualización en los protocolos de extracción dental en pacientes anticoagulados. Av. Odontoestomatol 2012; 28 (6): 311-320.

\section{INTRODUCCIÓN}

Se calcula que, en España, existen entre 600.000 y 700.000 pacientes en tratamiento con anticoagulantes orales, aumentando cada año en unos 100.000 . En el Reino unido alrededor de 300.000 personas están en tratamiento con anticoagulantes orales. Con la población en continuo envejecimiento en Europa y Norte América es un problema que preocupa y cada vez más en lo que a salud dental respecta (1). Una de las prestaciones básica cubiertas por la seguridad social en España así como una de las pres- 
taciones más frecuentes realizadas en las clínicas Odontológicas son las extracciones dentales simples o bien aquellas que impliquen algún acto quirúrgico a mayores como (incisiones, colgajos...).

Ante estos procedimientos quirúrgicos se han descrito varios protocolos en la literatura a fin de abordar médicamente a estos pacientes: suspensión del anticoagulante, reducción de la dosis, sustitución del principio activo e incluso el mantenimiento del fármaco anticoagulante (2).

\section{ANTICOAGULANTES ORALES}

Los anticoagulantes orales son antagonistas de la vitamina $\mathrm{K}$. La vitamina $\mathrm{K}$ es necesaria para la coagulación de la sangre dado que participa como cofactor en la síntesis de los factores de la coagulación II, VII, IX y X. La vitamina K se encuentra de forma natural y en cantidades importantes en vegetales de hoja verde y de forma farmacológica en fármacos como el (Konakion), de forma endógena ésta se consigue a expensas de la síntesis bacteriana en el tubo digestivo $(3,4)$.

Los anticoagulantes orales son también conocidos como antivitamina $\mathrm{K}$ (4) dado que su mecanismo de acción se basa en la inhibición de forma reversible del efecto de la vitamina $\mathrm{K}$, bloqueando, por tanto, la síntesis de los factores de la coagulación antes descritos. Podemos decir que los anticoagulantes orales (AO) actúan alargando el tiempo de coagulación de la sangre (5).

\section{FACTORES DE LA COAGULACIÓN (TABLA 1)}

De manera genérica definimos la hemostasia como "el mecanismo de defensa del organismo que tiene por objeto evitar la hemorragia, mantener la integridad de la pared vascular y restablecer la circulación sanguínea cuando se ha obstruido un vaso". Consta de cuatro etapas (7-11):

1. Vasoconstricción local.

2. Formación del trombo plaquetario: Adhesión y agregación.

3. Formación del trombo de fibrina: Coagulación.

4. Disolución del coágulo o fibrinólisis.

\section{TABLA 1.- NOMENCLATURA DE LOS FACTORES DE LA COAGULACIÓN}

\begin{tabular}{|c|l|}
\hline Número romano & Nombre \\
\hline I & Fibrinógeno \\
II & Protrombina \\
III & Tromboplastina \\
IV & Calcio \\
V & Proacelerina \\
VI & Igual que el factor V (este térmi- \\
& no se utiliza generalmente) \\
VII & Proconvertina \\
VIII & Globulina antihemofílica (AHG) \\
IX & Componente de la tromboplasti- \\
& na del plasma (PTC) \\
X & Factor Stuart-Prover \\
XI & Antecedente de la tromboplasti- \\
& na del plasma (PTA) \\
XII & Factor Hageman \\
XIII & Factor estabilizador de la fibrina \\
\hline
\end{tabular}

La coagulación sanguínea es por tanto una fase más del proceso de la hemostasia cuya finalidad radica en la transformación del fibrinógeno (proteína soluble que actúa como punto de unión interplaquetario en el trombo primario) en fibrina, insoluble, estable y resistente frente al sangrado (8).

Esta transformación tiene lugar a través de lo que conocemos como una activación enzimático proteica secuencial en cascada de una serie de componentes plasmáticos que de forma habitual se encuentran inactivos en la circulación. Ello sucede a través de dos vías la intrínseca y la extrínseca que a continuación de forma esquemática exponemos en la figura 1.

Según los datos publicados por industria farmacéutica en España los más vendidos son los derivados cumarínicos siendo el acenocumarol (sintrom) y la warfarina (aldocumar); la diferencia farmacológica principal entre ambos se encuentra en su farmacocinética, dado que el primero tiene una vida media plasmática más corta y por tanto su efecto puede revertirse con mayor rapidez (9).

Estos principios activos tienen como características principales: una buena absorción por vía oral, unión 


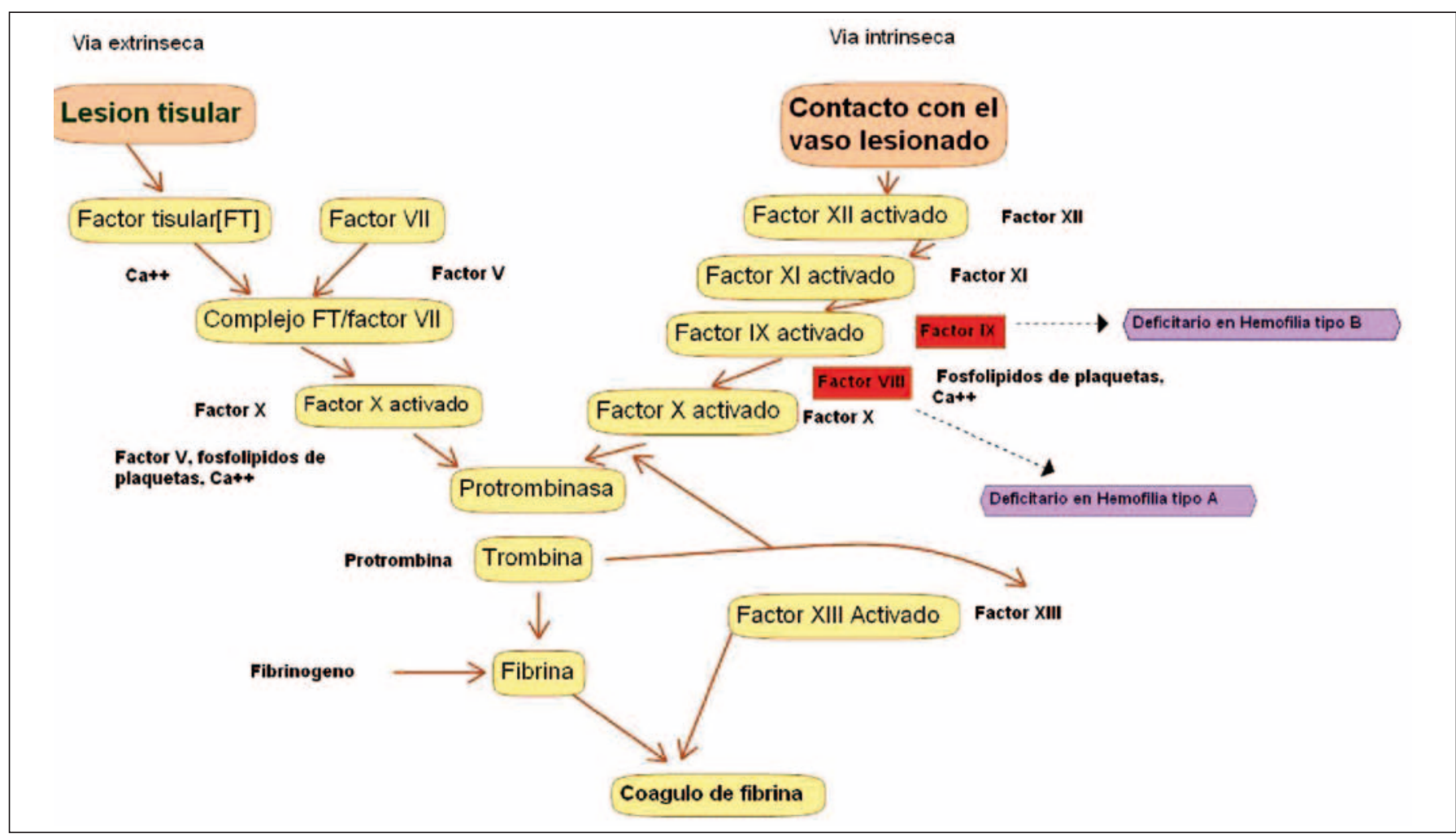

Fig. 1. Cascada de la coagulación.

a proteínas plasmáticas en más de un 97\%, metabolización hepática y excreción como metabolito inactivo vía urinaria. Tienen asimismo la característica de transplacentarios, atraviesan la barrera hematoencefálica (BHE) y pueden pasar a la leche materna.

Son muchos los fármacos que interactúan con los anticoagulantes orales y por tanto con el control del INR. (International Normalized Ratio) que es la proporción entre el tiempo de protrombina (TP) del paciente y un (TP) control. El INR, introducido en 1983, es el método de monitorización de la terapia ACO y está recomendado por la OMS como método para cuantificar de manera estándar los tiempos de protrombina. Normalmente el INR se mantiene en un rango de 2-3, a mayor INR mayor será la anticoagulación del individuo $(10,11)$.

En las tablas 2 a 6 se exponen los principales anticoagulantes orales, sus indicaciones y contraindicaciones, interacciones medicamentosas $(5,12)$.

Desde un punto de vista no farmacológico hay que destacar que: el consumo de alcohol, la disfunción

\section{TABLA 2.- ANTICOAGULANTES ORALES}

En la profilaxis de la trombosis, los fármacos más comúnmente usados son:

- Antiagregantes plaquetarios:

- Ticlopidina (Tiklid ${ }^{\circledR}$, Ticlodone $\left.{ }^{\circledR}\right)$

- Dipiridamol (Persantin ${ }^{\circledR}$, Miosen $\left.{ }^{\circledR}\right)$

- Trifusal (Disgren $\left.{ }^{\circledR}\right)$

- Ácido acetil salicílico (Adiro®, Tromalyt $\left.{ }^{\circledR}\right)$

- Clopidogrel (Plavix $\left.{ }^{\circledR}\right)$

- Cumarina y derivados (cumarínicofs):

- Acenocumarol (Sintrom $\left.{ }^{\circledR}\right)$

- Warfarina (Aldocumar $\left.{ }^{\circledR}\right)$

- Fenprocimona (Marcumar®)

- Heparina:

- Heparina de bajo peso molecular:

- Nadroparina (Fraxiparina ${ }^{\circledR}$ )

- Enoxaparina (Clexane $\left.{ }^{\circledR}\right)$

- Hiuridina recombinante:

- Desiuridina (Revasc $\left.{ }^{\circledR}\right)$

- Lepiuridina 


\section{TABLA 3.- INDICACIÓN DEL TRATAMIENTO CON ANTICOAGULANTES ORALES}

- Trombosis venosa profunda y tromboembolismo pulmonar.

- Prevención del embolismo de origen cardíaco:

- Valvulopatía mitral.

- Valvulopatía aótica.

- Prolapso de la válvula mitral.

- Fibrilación auricular no valvular crónica o paroxística.

- Fibrilación auricular y cardioversión electiva.

- Prótesis valvulares cardíacas mecánicas.

- Bioprótesis valvulares cardíacas.

- Infarto agudo de miocardio.

- Prevención secundaria del infarto agudo de miocardio.

- Miocardiopatía dilatada.

- Accidentes isquémicos transitorios repetitivos secundarios

- Enfermedad cerebrovascular o trombosis arterial cerebral progresiva.

- Disección de pared de la carótida intracraneal.

- Prevención de la oclusión tras cirugía reconstructiva arterial periférica.

hipertiroidismo), el ambiente cálido y la disminución de la ingesta de vitamina $\mathrm{K}$ en la dieta potencian el efecto de los anticoagulantes orales y por el otro lado lo disminuyen la ingesta de vitamina $\mathrm{K}$ en exceso, alcoholismo e hipotiroidismo (5-12).

Los principales efectos adversos de los anticoagulantes orales son las hemorragias, en particular aquellas que pueden producir daños irreversibles como por ejemplo la hemorragia intracraneal o de cualquier estructura que produzca compresión de órganos vitales así como de otras que no puedan diagnosticarse de forma inmediata como son las hemorragias internas.

\section{PROTOCOLOS MÉDICOS DE LAS PRINCIPALES ÁREAS DE SALUD DESCRITOS EN LA LITERATURA}

El departamento de Sanidad y Seguridad social de Cataluña (12) en la descripción del protocolo de
TABLA 4.- CONTRAINDICACIONES DEL TRATAMIENTO ANTICOAGULANTE ORAL

\section{Contraindicaciones absolutas:}

- Diátesis hemorrágicas graves.

- Alergia al medicamento.

- Procesos hemorrágicos activos incluyendo:

- Ulcus sangrante.

- Neoplasias ulceradas.

- Retinopatía hemorrágica.

- Hemorragia intracraneal reciente.

- Aneurisma intracerebral.

- Hipertensión arterial grave o no controlable.

Contraindicaciones relativas:

— Mala absorción intestinal.

- Ulcus gastroduodenal activo.

- Enfermedad hepática o renal severa.

- Cirugía reciente del sistema nervioso central.

- Esteatorrea.

- Retinopatía hemorrágica.

- Alcoholismo activo.

- Alteraciones de la marcha.

- Escaso nivel mental.

- Alteración mental, falta de colaboración.

— Trastornos psiquiátricos con tendencia al suicidio.

— Epilepsia.

- Pericarditis con derrame.

- Pacientes con pronóstico vital infausto a muy corto plazo.

— Primer trimestre y último mes en gestación.

actuación para el control de pacientes con tratamiento anticoagulante oral recomienda para la realización de extracciones dentales:

1. Comprobación del INR previo a la extracción dental y su valoración dentro de unos márgenes o rangos terapéuticos.

2. Maniobras Hemostáticas postoperatorias con la utilización de ácido tranexámico durante veinte minutos y compresión con gasa en la zona intervenida.

La consejería de salud del SAS (13) (servicio andaluz de salud) establece el siguiente protocolo:

1. El mismo día de la extracción se realizar un control del nivel de anticoagulación para asegurar que está dentro del rango terapéutico $(\mathrm{INR}<3)$ 


\begin{tabular}{l} 
TABLA 5.- INTERACCIONES \\
MEDICAMENTOSAS DE LOS \\
ANTICOAGULANTES ORALES. FÁRMACOS \\
QUE INTERFIEREN CON MAYOR CONSTANCIA \\
E INTENSIDAD \\
\hline Potenciadores: \\
- Analgésicos-antiinflamatorios: \\
- Ácido acetilsalicílico. \\
- Antimicrobianos y antiparasitarios: \\
- Cotrimoxazol. \\
- Tetraciclinas. \\
- Imidazoles. \\
- Hipovolemiantes: \\
- Fibratos. \\
- Varios: \\
- Amiodarona. \\
Inhibidores: \\
- Rifampicina. \\
— Barbitúricos. \\
— Fenitoina. \\
— Colestiramina. \\
— Colestipol. \\
— Poliestireno sulfonato cálcico. \\
— Carbamacepina. \\
— Suplementos dietéticos que aporten vitamina K. \\
\hline
\end{tabular}

2. En caso de requerir profilaxis de endocarditis, ésta se pautará de forma convencional siguiendo el protocolo médico de la sociedad americana de cardiología.

3. Después de la exodoncia se irriga la zona con una ampolla de 500 mg de ácido tranexámico (Amchafibrin $\left.{ }^{\circledR}\right)$; se recomienda la aplicación de puntos de sutura. Compresión activa en la zona con gasa empapada 20 minutos

4. Enjuagues cada 6 horas durante 2 días con ácido tranexámico sin tragar.

5. No ingesta de alimentos duros ni calientes durante las primeras $48 \mathrm{H}$.

La sociedad Valenciana de medicina familiar y Comunitaria (14) recomienda no suspender los ACO para una extracción dental si y siempre que el INR del paciente se encuentre entre $2-4$; repetir las maniobras postoperatorias similares que en los dos casos anteriores; no prescribir fármacos antiinflama-

\section{TABLA 6.- FÁRMACOS QUE INTERFIEREN DE FORMA INCONSTANTE O HABITUALMENTE CON MENOS INTENSIDAD}

\section{Potenciadores:}

- Analgésicos-antiinflamatorios:

- Sulfinpirazona

- Indometacina

- Naproxen

- Dipironas

- Piroxicam

- Antimicrobianos y antiparasitarios:

- Macrólidos

- Quinolonas

- Isoniacida

- Clindamicina

- Hipovolemiantes:

- Simvastatina y ocasionalmente otros inhibidores de HMG-CoA

- Psicofármacos:

- Antidepresivos tricíclicos

- Paroxetina, fluoxetina

- Clorpromacina

- Varios:

- Clorpropamida y otras sulfonilureas

- Antiulcerosos (cimetidina, ranitidina, omeprazol, ansoprazol)

- Tiroxina

- Tamoxifeno

- Disulfiran

Inhibidores:

- Aminoglutetimida

- Ciclosporina A

- Ticlopidina

— Ritonavir

torios no esteroideos no selectivos ni inhibidores de la COX-2. Asimismo preconizan el uso de esponjas hemostáticas, y suturas en todos los casos.

En los protocolos consultados del servicio Canario de Salud, (15) establecen la siguiente pauta:

- Si INR $<2$ no modifican la pauta.

- Si INR2-2,5 indican la no ingesta del medicamento la noche antes de la intervención.

- Si INR >2,5 establecer un tratamiento puente con heparina o disminuir más aun el ACO. 
La sociedad española de Cirugía Oral y Maxilofacial recomienda, en aquellas cirugías limitadas a menos de tres extracciones dentales, no modificar el tratamiento con ACO siempre que el INR sea menor a 3,5 y sin otros factores moduladores del riesgo (entre otras interacciones medicamentosas, patologías...).

Los protocolos revisados por el SESCAM $(16,17)$ en relación a esta materia se basan en revisar el INR 7 días previos a la intervención para evaluar su situación dentro de los márgenes terapéuticos; se suspende éste el día anterior a la intervención y será atendido con HBPM en la tarde antes de la intervención en casos de alto riesgo tromboembólico.

\section{REVISIÓN DE LOS PRINCIPALES ESTUDIOS DE MANEJO DE PACIENTES EN TRATAMIENTO (ACO)}

Autores, como Devani, Campbell y Beirne (18-20), estudiando la warfarina como ACO en un grupo de pacientes con unos INR 3-4 no encuentran diferencias significativas entre pacientes, a los que se les ha interrumpido la terapia anticoagulante y a los que no. Concluyen que si el tratamiento quirúrgico se prolongase o fuese más agresivo tendrían que revisar dichos ACO.

A los estudios de los anteriores se suman los descritos por Ramstrom (21) con valores de INR entre 2 y 4 y Blinder (22) con rango de 1,5 a 3,5.

Souto en su estudio prospectivo randomizado con pacientes anticoagulados y con INR en rango de 24 tampoco observa diferencias estadísticamente significativas (23).

Bodner (24) utilizó en su estudio sobre la eficacia de sellantes de fibrina en pacientes anticoagulados con distintos rangos de INR unos adhesivos de fibrina con los que concluyó que no existía relación entre INR, trauma quirúrgico y sangrado.

Autores como los anteriores y otros, como Webster y Scully, son partidarios de no modificar las pautas de tratamientos con ACO y potenciar las medidas procoagulantes locales así como los procedimientos posquirúrgicos necesarios para llevar a cabo después del tratamiento oral. Scully, recomienda realizar la cirugía por la mañana a fin de tener más tiempo durante todo el día para vigilar la hemostasia $(25,26)$.

Autores, como Visintini, establecen, en sus estudios sobre extracciones dentales en pacientes en tratamiento con anticoagulantes orales y revisando la literatura en 2006, un mantenimiento del fármaco ACO así como una sustitución de éste por heparina de bajo peso molecular en aquellos casos en los que se vayan a realizar intervenciones más complejas (27).

Cerezuela (5) y cols. en su guía clínica de cirugía bucodental ambulatoria en pacientes con terapia anticoagulante oral (2007) recomienda no suspender el ACO siempre que no vayan a realizarse procesos agresivos o invasivos y siempre que presenten un INR $<3$. excepto en pacientes portadores de prótesis valvulares cardíacas en los que se acepta un INR $<3,5$. Asimismo resalta una metodología clínica para abordar estos pacientes de la forma menos traumática posible, con la aplicación de ácido tranexámico irrigado en la zona e incluso introducir alguna esponja de fibrina o celulosa oxidada, en el lecho alveolar; asimismo destaca la necesidad de suturar la herida siempre que ésta sea posible. En relación a la aplicación de antifibrinolíticos como es el ácido tranexámico, derivado sintético de la lisina que se une a los lugares de fijación de la misma en las moléculas de plasminógeno y plasmita bloqueando así de forma reversible esta unión e inhibiendo la fibrinólisis. Su uso ha sido demostrado como muy satisfactorio en múltiples estudios entre los que destacamos los de Borea, Ramstrom y Sindet-Pedersen. (1991-1993), con la ventaja de no haber demostrado efectos sistémicos con su uso local. Cabe destacar que su uso no está aun legislado en algunos países como EEUU 7 Reino unido cuyo sustituto molecular es el ácido Epsilon aminocaproico (Caproamin $\left.{ }^{\circledR}\right)$ al $25 \%$ (250 mg/ml) en enjuagues con 5-10 ml $(8,27,28)$.

\section{DISCUSIÓN}

De la información anteriormente expuesta se deduce en relación al manejo de los pacientes anticoagulados en el gabinete dental que el INR es un método 
sencillo y estandarizado que es usado de forma rutinaria por la mayoría de los Odontólogos y cirujanos maxilofaciales para controlar y analizar el nivel de anticoagulación en este tipo de pacientes y ante procedimientos quirúrgicos orales (29).

Existe cierta limitación sobre los estudios de pacientes con terapia ACO en base a la falta de consensos y a la controversia que el tema genera. En general las recomendaciones y normas de actuación deben basarse en varios conceptos tales como los estudios publicados, el sentido común , la evidencia clínica y el fácil acceso a las recomendaciones (30-34).

Por un lado nos encontramos a autores como Tinker, Kearon y Hirsch que justifican una interrupción mínima de la terapia ACO en base a un mínimo riesgo tromboembólico y alto riesgo de complicaciones por sangrado. Son muchos los autores que en sus estudios no han encontrado ninguna complicación tromboembólica, pero sí de sangrado posquirúrgicos, Eckman, Butler y Saour (7, 28, 33).

En sentido contrario, nos encontramos con múltiples autores, en los que no ponen de manifiesto ningún episodio de sangrado relevante en pacientes con terapia ACO y sí con complicaciones embólicas en aquellos en los que se suspendió el tratamiento ACO. Los estudios de Scully (26) consideran que la mayoría de los procesos hemorrágicos producidos en este grupo de pacientes se debieron a otros procesos diferentes del tratamiento farmacológico, tales como: cirugía agresiva sin mínimo trauma quirúrgico, escaso seguimiento por parte del paciente de las recomendaciones posquirúrgicas, hipertensión e interacciones farmacológicas entre otras). Wahls describió 12 casos sobre 2.400 intervenciones medidas extra para el control del proceso hemorrágico (34-36).

Como protocolo híbrido entre ambas vertientes en el manejo de estos pacientes se encuentra la aplicación de un protocolo de sustitución, preferido por un $65 \%$ de los hematólogos españoles, así como guía de recomendación de la American Collage of Cheste Physicians (37-39). En aquellos casos en los que haya que seguir un protocolo para la iniciación y modificación de la terapia con heparina seguiremos las siguientes directrices descritas por Mehra (40) en el JOMS a propósito de una revisión de protocolos.

\section{CONCLUSIONES}

En base a la literatura y protocolos revisados en relación al manejo clínico- farmacológico de pacientes en tratamiento ACO podemos establecer que la pauta terapéutica a seguir dependerá de los siguientes aspectos:

1. Control del INR del paciente y el tipo de cirugía a realizar.

2. Vigilancia y cumplimiento de las normas posquirúrgicas.

3. Interacciones farmacológicas.

4. Riesgo tromboembólico (moderado, media o alto).

5. Otras patologías coexistentes que puedan aún más alterar la hemostasia.

Integrando los conceptos anteriores llevaremos a cabo un protocolo de manejo del paciente ACO de una manera u otra, aunque la tendencia de los protocolos actuales y de los estudios clínicos aconsejan, siempre que nos encontremos dentro de un INR en rango terapéutico y que tanto el proceso quirúrgico como la patología de base del paciente así lo permitan: no modificaremos la pauta farmacológica ACO.

En estos casos será fundamental llevar a cabo las siguientes normas por parte del profesional:

La realización de un procedimiento quirúrgico lo más mínimamente invasivo y lo más relajante para el paciente a fin de evitar la ansiedad en el tratamiento.

Por otro lado nos ayudaremos de agentes hemostáticos locales; de forma intraoperatoria y en forma de enjuague postoperatoria; el más utilizado en nuestro país es el ácido tranexámico.

Recomendar otras alternativas terapéuticas al proceso quirúrgico si fuesen éstas viables tales como obturaciones, endodoncias...

Realizar la cirugía a primera hora de la mañana a fin de poder atender cualquier complicación durante el día.

Uso de anestésicos con vasoconstrictor siempre que sea posible y técnicas anestésicas infiltrativas no tronculares. 
Medidas hemostáticas postoperatorias tales como el Surgicel ${ }^{\circledR}$, la aplicación de puntos de sutura, compresión hemostática duradera con una gasa impregnada en ácido tranexámico...

Control de la farmacología prescrita de forma pre y postoperatoria dado que ésta pueda alterar potenciando o restando actividad farmacológica a los ACO.

Evaluación y control del cumplimiento terapéutico del paciente: Dieta blanda y fría las primeras 24-48 Horas; enjuagues con antifibrinolítico (ácido tranexámico 4,8-5\% o ácido épsilon amino caproico $25 \%$ ).

\section{AGRADECIMIENTOS}

El autor, Antonio Bascones Martínez, ha recibido un proyecto de Investigación de Fundación Mutua Madrileña (AP 87102011) con cuya ayuda se ha realizado el presente trabajo.

\section{BIBLIOGRAFÍA}

1. Webster K, Wilde J. Management of anticoagulation in patients with prosthetic heart valves undergoing oral and maxillofacial operations. $\mathrm{Br} \mathrm{J}$ Oral Maxillofac Surg 2000;38:124-6.

2. Giner J, González A, Ordóñez V, Martín-Granizo $\mathrm{R}$, Berguer A. Actualización de la cirugía oral en el paciente anticoagulado. Rev Esp Cirug Oral y Maxilofac 2003;25:294-303.

3. Wahl MJ. Dental surgery in anticoagulated patients. Arch Intern.Med 1998;158:1610-6.

4. Silvestre F, Plaza A. Odontología en pacientes especiales. $1^{a}$ Edición. Universidad de Valencia. 2007.ISBN 8437066522.

5. Cerezuela E, Pérez A, Tejedor A, Ramos B. Guía clínica de cirugía bucodental ambulatoria en pacientes con terapia anticoagulante oral. Cartagena, 2007.

6. Halfpenny W, Fraser J, Adlam D. Comparison of 2 hemostatic agents for the prevention of postextration hemorrhage in patiens on anticoagulants. Oral Surg Oral Med Oral Pathol Oral Radiol Endod 2001;92:257-9.

7. Saour JM, Ali HA, Mammo LA y cols. Dental procedure in patients receiving oral anticoagulation therapy. J Heart Valve Dis.1994;3:315-7.

8. Ramström G, Sindet-Petersen S, Hall G et al. Prevention of postsurgical bleeding in oral surgery using tranexamic acid without dose modification of oral anticoagulants. J Oral Maxillofac Surg 1993; 51:1211-16.

9. Todd D, Roman A. Outpatient use of low-molecular weight heparin in an anticoagulated patient requiring oral surgery: case report. J Oral Maxillofac Surg 2001;59: 1090-2.

10. Majerus P, Dalcette B, Hermans M, Pourtois M, Capel P. Variations in fibrinolytic activity of human whole saliva. Eur J Oral Sci 1996;104:341-5.

11. Souto JC, Oliver A, Zuazu-Jausorol et al. Oral surgery in anticoagulated patients without reducing the dose of oral anticoagulant: a prospective randomised study. J Oral Maxillofac Surg 1996;54:27-32.

12. Guía de actuación en pacientes odontológicos anticoagulados en Atención Primaria J.M. Rodríguez Zafra, J.A. Casero Nieto JADA, Vol. 5 No 6 Diciembre 2010.

13. Guía para el paciente anticoagulado. 2005 Servicio Andaluz de Salud. Consejería de Salud. Junta de Andalucía.

14. Como programar en un paciente anticoagulado/ antiagregado la extracción dental. Societat valenciana de medicina comunitaria y familiar. 2008.

15. Criterios a seguir en la atención en pacientes con coagulopatías y con tratamiento con anticoagulante. Pautas de actuación. Programa de salud oral en atención primaria dirección general de programas asistenciales servicio canario de la salud. 2010. 
16. Rotaeche R, Etxeberria A, Moreno M. Conducta a seguir en las extracciones dentales en los pacientes que toman aspirina y clopidogrel como profilaxis secundaria de enfermedad cardiovascular [Internet]. A Coruña: Fisterra.com; 2007.

17. Altirriba J, Mar-Ballestes M, Vila Duart F, et. al. Anticoagulación oral. FMC 2004;11(Supl 1):20-2.

18. Devani P, Lavery KM, Howell CJ. Dental Extractions in patiens on Warfarin: is alteration of anticoagulant regimen necessary? J Oral Maxillofacial Surg 1998;36:107-11.

19. Campbell JH, Alvarado F, Murray RA. Anticoagulation and minor oral surgery: should the anticoagulation regimen be altered? J Oral Maxillofac Surg 2000;58:131-5.

20. Beirne OR, Koehler JR. Surgical manegnement of patients on warfarin sodium. J Oral Maxilofac Surg 1996;54:1115-8.

21. Ramstrom G, Sindet-Pedersen S, Hall G, Blomback $M$, Alexander U. Prevention of postsurgical bleeding in oral surgery using tranexamic acid without dose modification of oral anticoagulants. J Oral Maxillofac Surg 1993;51: 1211-6.

22. Blinder D, Manor Y, Martinowitz U, taicher S. Dental extractions in patients maintained on oral anticoagulant therapy: comparison of INR value with occurrence of postoperative bleeding. Int $\mathrm{J}$ Oral Maxillofac Surg 2001;30:518-21.

23. Souto JC, Oliver A, Zuazu-Jausorol y cols. Oral surgery in anticoagulated patients without reducing the dose of oral anticoagulant: a prospective randomised study. J Oral Maxillofac Surg 1996;54:27-32.

24. Bodner L, Weinstein JM, Baumgarten AK. Efficacy of fibrin sealant in patients on various level of oral anticoagulant undergoing oral surgery. Oral Surg Oral Med Oral Pathol Oral Radiol Endod 1998;86:421-4.

25. Kakkar V, Howes J, Sharma V, Kadziola Z. A comparative, double blind, randomized trial of a new second generation LMWH and UFH in the prevention of pos302 Rev Esp Cirug Oral y Maxilofac 2003;25-9a.

26. Scully C, Cawson RA. Medical problems in dentistry. 4th ed. Oxford, London and Boston: Wright; Butterworth-Heinemann, 1997.

27. Borea G, Montebugnoli L, Capuzzi P et al. Tranexamic acid as a mouthwash in anticoagulant-treated patients undergoing oral surgery. Oral Surg Oral Med Oral Pathol Oral Radiol Endod 1993;75:29-31.

28. Eckman $M H$, Beshansky JR, Durand-Zaleski I, Levine HJ, Pauker SG. Anticoagulation for noncardiac procedures in patients with prosthetic heart valves: does low risk mean high cost?. J Am Med Assoe 1990;263:1513-21.

29. Spandorfer J, Merli G, Lowson SM y cols. Anticoagulation and elective surgery (2). N Engl J Med 1997;337:938-40.

30. Mehra P, Cottrell DA, Bestgen SC, Booth DF. Management of heparin therapy in the high-risk, chronically anticoagulated, oral surgery patient: a review and a proposed nomogram. J Oral Maxillofac Surg 2000;59:198-202.

31. Schardt-Sacco D. Update on coagulopathies. Oral Surg Oral Med Oral Pathol Oral Radiol Endod 2000;90:559-63.

32. Mismetti P, Laporte-Simitsidis S, Tardy B y cols. Prevention of venous thromboembolism in internal medicine with unfractionated of low molecular weight heparins:a meta-analysis of randomized clinical trials. Thromb Haemost 2000;83:14-9.

33. Buttler AC, Tait RC. Management of oral anticoagulant induced intracranial haemorrhage. Blood Rev 1998;12:35-44.

34. Wahl MJ. Dental surgery in anticoagulated patients. Arch Intern Med 998;158:1610-6.

35. Katholi RE, Nolan SP, McGuire LB. Living with prosthetic hesrt valves: subsequent non-cardiac 
operations and the risk of thromboembolism or haemorrage. Am Heart J 1976;92:162-7.

36. Wahl MJ. Myths of dental surgery in patients receiving anti-coagulant therapy. J Am Dent Assoc 2000;131:77-81.

37. Poller L, Tomson J. Evidence for «rebound» hypercoagulability after stopping anticoagulants. Lancet 1964;ii:62-4.

38. Ad Hoc committee of the Working Group on Valvular Heart Disease. European Society of Cardiology. Guidelines for the prevention of thromboembolic events in valvular heart disease. J Heart Valve Dis 1993;2:398-410.
39. Diz Dios P, Fernández Feijoo J. Tooth removal and anticoagulant therapy.. Oral Surg Oral Med Oral Pathol Oral Radiol Endod 2001;92 (3):248-9.

40. Mehra P, Cottrell DA, Bestgen SC, Booth DF. Management of heparin therapy in the high-risk, chronically anticoagulated, oral surgery patient: a review and a proposed nomogram. J Oral Maxillofac Surg 2000;59:198-202.

\section{CORRESPONDENCIA}

J. Ripollés de Ramón

Platerías, 1

28016 Madrid. 